\title{
AMBITOS DE APLICACIÓN DE LA TEORÍA DE LA MODIFICABILIDAD ESTRUCTURAL COGNITIVA DE REUVEN FEUERSTEIN.
}

\author{
SCOPES OF APPLICATION OF REUVEN FEUERSTEIN'S STRUCTURAL \\ COGNITIVE MODIFIABILITY THEORY.
}

\author{
Recibido: febrero de 2013 - Revisado: agosto de 2013 - Aceptado: 30 de octubre de 2013
}

Por: Abad E. Parada-Trujillo ${ }^{1}$, William R. Avendaño C. ${ }^{2}$

\section{RESUMEN.}

En el artículo se hace una exploración de la teoría de la Modificabilidad Estructural Cognitiva (MEC) de Reuven Feuerstein, en relación con los ámbitos de aplicación de la misma. Para lo anterior, se ha hecho una recopilación de algunos estudios realizados en América Latina, Estados Unidos y Europa, a fin de identificar los diversos escenarios en que la teoría puede tener aplicación. Los resultados evidencian que la teoría de Feuerstein está ligada al concepto de desarrollo humano y que los sistemas creados con base en la MEC permiten reducir la deprivación cultural de muchas personas sin importar los factores endógenos que le afecten.

\section{PALABRAS CLAVES.}

Modificabilidad Estructural Cognitiva, Feuerstein, deprivación cultural, inteligencia, cognición.

\begin{abstract}
.
An exploration of Reuven Feuerstein's cognitive structural modifiability (CSM) theory, in relation to the scope of its application, is made in the article. For this reason, a compilation of studies in Latin America, The United States, and Europe, is made, in order to identify the different scenarios in which the theory can be applied. The results show that Feuerstein's theory is linked to the concept of human development and that the systems created, based on the CSM, allow the reduction of the cultural deprivation of many people, regardless of the endogenous factors that affect it.
\end{abstract}

\section{KEY WORDS AND EXPRESSIONS.}

Structural cognitive modifiability theory; Feuerstein; Cultural deprivation; Intelligence; and Cognition.

${ }^{1}$ Abogado (Universidad Libre, Colombia), Especialista en Alta Gerencia (Universidad Libre, Colombia), Magister en Educación (Universidad Externado de Colombia, Colombia). Docente-Directivo Colegio Militar General Francisco de Paula Santander (Cúcuta, Colombia. e-mail: abadernesto@hotmail.com.

${ }^{2}$ Economista en Comercio Exterior (Universidad Los Libertadores, Colombia), Especialista en Alta Gerencia (Universidad Libre, Colombia), Magister en Administración (Universidad Santo Tomas, Colombia) y Doctorando en Ciencias Sociales y Humanas (Pontificia Universidad Javeriana, Colombia). Profesor adscrito al Departamento de Estudios Internacionales y de Fronteras (Facultad de Ciencias Empresariales), Universidad Francisco de Paula Santander (Cúcuta, Colombia). Director del Grupo de Investigación (GIRS) en Responsabilidad Social. e-mail: wrac2008@hotmail.com. 


\section{Introducción.}

En el campo de la acción pedagógica el maestro debe atender a las características físicas, psíquicas, emocionales, socio-históricas, politicas, etc., de los sujetos de aprendizaje. Por ejemplo, elementos de esencial reflexión para los maestros en su acción pedagógica es la pobreza, la inequidad y la miseria como estados de vulneración. Estos factores endógenos (genéticos, biológicos, emocionales, culturales) influyen de forma considerable en la capacidad o incapacidad de los sujetos para responder de forma adecuada y precisa a los estímulos. Tanto maestros como otros profesionales que tienen por función potenciar el desarrollo humano de los individuos, ostentan la tarea de reflexionar en torno a cada uno de los factores de exclusión a fin de generar mejores estados tanto en niños y niñas, como en jóvenes y adultos.

Debido a la complejidad del proceso formativo, múltiples corrientes se han consolidado a fin de explicar los procesos de enseñanza-aprendizaje o de formación. De este modo, el constructivismo (Piaget e Inhelder, 2007), el aprendizaje significativo (Ausubel, 1976), la teoría sociocultural e histórica (Vigotsky, 1975), las inteligencias múltiples (Gardner, 1985), entre otras, ofrecen supuestos epistemológicos que permiten la comprensión de los procesos de enseñanza y aprendizaje, el desarrollo humano y el crecimiento personal. Otra teoría es la formulada por Reueven Feuerstein: Modificabilidad Estructural Cognitiva (MEC), la cual tuvo un origen especial y ha sido posicionado como una teoría vanguardista e innovadora por la esperanza que imprime a las vidas de las personas, a pesar de las condiciones en que se encuentre. En Colombia y América Latina se han realizado algunos estudios con base en la teoría de la MEC, la mayoría de tipo descriptivo y exploratorio, y algunos con aplicación de algunos de los programas de la teoría. Sin embargo, la revisión de la literatura sobre la teoría de la MEC evidencia una aplicación diversa y rica de la teoría, que permite abrir nuevos campos de uso con grupos poblacionales con diferentes afectaciones.

Tradicionalmente, las contribuciones de la teoría de la MEC, han sido empleadas en el campo educativo, permitiendo que se replantee el papel del maestro como transmisor de la información, las relaciones verticales de los sujetos de enseñanza-aprendizaje, el aprendizaje a través de la repetición y el rol pasivo del educando, y que ha sido afianzado por los modelos heteroestructurantes (De Zubiría, 1997). En efecto, hay una fuerte crítica a los sistemas educativos como el colombiano, en el que se ha desconocido los factores sociales, económicos, políticos y culturales de los educandos. De allí que Monclús (2004, p. 11) describa que la educación ha caído en modelos y esquemas que se han repetido de forma generalizada, concretándose en currículos formales, planos y angostos (Pilonieta, 2004). La teoría de la $\mathrm{MEC}$ ofrece nuevos escenarios de análisis a partir de tres sistemas creados con base en la misma teoria: (i) el Programa de Enriquecimiento Instrumental (PEI), el Diagnóstico Dinámico del Potencial de Aprendizaje (LPAD) y los Ambientes Modificantes.

\section{Metodologia.}

El artículo presentado tiene por objetivo hacer una exploración de la teoría de la MEC a partir de sus conceptos básicos y analizar los alcances de la misma a través de los diversos estudios e investigaciones que han tenido como base los aspectos esenciales de la teoría. En otras palabras, el artículo busca determinar los escenarios de aplicación de la teoría así como la validez de sus aportes a comunidades afectadas o excluidas. A continuación se explica de forma breve el origen de la teoría de la MEC, los elementos básicos que la integran y, por 
último, una revisión de los estudios que demuestran la variedad de aplicaciones. Para lo anterior, se ha hecho un rastreo de los diversos estudios e investigaciones, los cuales han sido analizados y sintetizados a fin de dar cumplimiento del objetivo central.

\section{Resultados.}

La teoría de la mec y conceptos básicos

Human beings have the unique characteristic of being able to modify themselves no matter how they start out. Even inborn barriers and traumas can be overcome with belief and the right mediation. Jackson y Feuerstein (2011, p. 55)

La MEC es una teoría formulada y desarrollada por el rumano Reuven Feuerstein, Doctor de la Universidad Sorbona de Paris (Francia) y quien realizó estudios de psicología (1944), psicología clínica (1952) y psicología del desarrollo (1970). Desde una muy corta edad comenzó su labor de maestro (ocho años) con jóvenes en promedio de 14 años, a quienes debía enseñar a leer y a escribir. Su trabajo científico inició en la postguerra con los niños, niñas y jóvenes sobrevivientes del Holocausto, quienes quedaron con problemas de adaptación y de aprendizaje. El trabajo con estas personas le permitió a Feuerstein verificar el cambio o la modificación, razón por la que empezó a buscar y elaborar el respaldo epistemológico que sustentará sus hipótesis.

El resultado de la labor desarrollada llevó a Feuerstein a elaborar el siguiente principio, base de su teoría y de toda las investigaciones realizadas durante las últimas décadas: "el organismo humano es un sistema abierto que en su evolución adquirió la propensividad para modificarse a sí mismo, siempre y cuando exista un acto humano mediador" (Feuerstein, 1963). Esta posición es de tipo revolucionaria al considerar que pese a cualquier condición de la persona existe la posibilidad de transformación. La teoria de la MEC de Feuerstein ha tenido un importante impacto y ha despertado el interés generalizado de la comunidad académica y científica. Resultado de este auge es la aplicación de la teoría en más de 70 países y su validación en diversos contextos y grupos poblacionales. La teoría de la MEC está inscrita en las ciencias del comportamiento y en el ámbito cognitivo, y cuyo objetivo es propiciar nuevos estados en las personas de tipo comportamental, emocional y cognoscitivo.

La teoría MEC defiende tres grandes ideas. En primer lugar, el ser humano no sólo es el resultado de un proceso de evolución biológica sino que sumada a esta condición se encuentra la esfera socio-cultural de la cual también es producto. En segundo lugar, la teoría en cuestión considera el comportamiento como un estado, una manifestación de lo que a nivel de pensamiento está sucediendo. Por último, los avances en la neurociencia le han brindado un estatus científico sólido a la teoría de la MEC además de las experiencias significativas en donde se ha implementado la teoria. Entonces, no es raro considerar los aportes de Feuerstein y su teoría como verdaderos logros con impactos sobresalientes, como lo describe Hobbs (1980, p. 566).

\section{- La inteligencia}

La inteligencia de acuerdo a los estudios realizados en el campo de la neurociencia y otras ciencias y disciplinas, tiene relación con la actividad mental del sujeto y la capacidad del mismo. Por ejemplo, Myers (2005, p. 423) la describe como una "cualidad mental que consiste 
en la capacidad para aprender de la experiencia, resolver problemas, y utilizar el conocimiento para adaptarse a las situaciones nuevas". La inteligencia desde los aportes de Myers (2005) se concibe como una capacidad de uso adecuado del conocimientos para resolver problemas que le permitan a la persona adaptarse a las situaciones, es decir, que su reflejo se encuentra en la práctica social (Suazo, 2006. p. 15).

Para Gardner (1985) la inteligencia se refiere a un conjunto de habilidades o aptitudes mentales, la cual posee todos los sujetos en diferentes niveles y que le permite "elaborar productos que sean valiosos en una o más culturas". Al igual que Myers (2005), Gardner (1985) asume la inteligencia como una capacidad de adaptación, misma ruta elaborada por Piaget (1980, p. 4).

Ahora bien, al explicar el concepto de inteligencia y hacer una relación entre esta y la MEC, Feuerstein (1980) y Feuerstein, et. al. (1988) advierten que la inteligencia no es estática sino que esta es dinámica y modificable, y esta potencialidad que tenga el sujeto para hacerlo en términos de adaptación es lo que se denomina inteligencia:

[...] la inteligencia (es) un proceso en lugar de una entidad fija, inmutable y concreta. Proceso lo bastante amplio como para abarcar una enorme variedad de fenómenos que tienen en común la dinámica y la mecánica de la adaptación, en su acepción más genérica; es decir los cambios que sufre el organismo como respuesta a la aparición de una nueva situación que requiere tales cambios. Esta adaptabilidad del organismo es a la que nos referiremos por modificabilidad. Esta propensión al cambio, esta flexibilidad y plasticidad, es la que entenderemos como inteligencia. (Feuerstein, 1980)

La inteligencia desde la teoría de la MEC excluye como posibilidad la medición de esta capacidad a través de las pruebas de coeficiente intelectual, pues considera que esto "hace retroceder más de 50 años, haciendo caso omiso de todos los logros de la psicología cognitiva y del desarrollo en la última mitad del siglo XX" (Feuerstein y Kozulin, 1995. p. 72). Para Feuertein y Kozulin (1995) la inteligencia, es decir, el poder intelectual del organismo humano depende de innumerables variables de tipo biológicas, sociales y culturales.

- Deprivación cultural.

La deprivación cultural es una condición que se caracteriza por la "baja capacidad de los individuos para ser modificado a través de la exposición directa a los estímulos, una condición causada por la falta de Experiencias de Aprendizaje Mediado (EAM)" (Haywood y Tzuriel, 1992. p. 235). La manera como se manifiesta la deprivación cultural es a través de las deficiencias en las funciones cognitivas las cuales pueden corregirse a través de la EAM.

Feuerstein y Jackson (2011, p. 57) incluyen el término cultura en este binomio pues consideran que "la cultura es compartida y elaborada a través de interacciones seleccionadas específicamente por un padre, pariente o maestro (a una persona que él llamó un mediador) para ampliar el conocimiento y desarrollar las capacidades intelectuales de un niño", así cuando los sujetos "están desconectados de estas interacciones culturales, hay un corto circuito en las formas de pensar que les permitan dar sentido, interpretar, inferir o, lo que limita su desarrollo intelectual y capacidad de adaptación" (Jackson y Feuerstein, 2011. p. 57). En otras palabras, las construcciones culturales o los contextos culturales dan sentido al conocimiento y a la adaptación del sujeto al medio. 


\section{- La Experiencia de Aprendizaje Mediado (EAM).}

La EAM constituye el principal factor causa en la modificación de los sujetos y la cual está definida por la calidad de la interacción entre el organismo humano con su medio ambiente. Feuerstein (2002) en entrevista realizada por Noguez (2002) menciona que la EAM "conlleva a la explicación de los procesos cognoscitivos como subproducto de la transmisión cultural": [...] la interacción del niño con un humano es un factor clave en el desarrollo humano. La EAM es una de las dos modalidades de interacción mediante la cual se produce el desarrollo cognitivo a partir del cambio o la interacción del organismo con el medio ambiente. La otra modalidad es la exposición directa e inmediata del niño a las fuentes de estímulos. Evidentemente, el énfasis en la cantidad enriquecido y la variación en los estímulos ambientales se supone que afectan el desarrollo cognitivo y el cambio. La relación directa entre el estímulo y la respuesta del niño (SR) es tal vez sus raíces en las teorías influyentes del conductismo. (Feuerstein, et. al. 1994. p. 300)

La EAM representa una de las modalidades para el aprendizaje de las personas. Estas dos son: la exposición directa a los estímulos (corresponde al modelo conductista) y la mediación en la interacción entre ambiente y sujeto. La siguiente figura representa la EAM de Feuerstein, en donde se destaca el organismo en el centro, el cual está expuesto a los estímulos sobre los cuales media el maestro (o cualquier otro sujeto), y como resultado de ello se produce una respuesta, la cual es tomada por el mediador para impulsar el cambio respectivo en el sujeto de aprendizaje. En la siguiente figura se representan los tres modelos de aprendizaje clásicos: el conductista, el constructivista y la EAM.

\section{Figura 1.}

Experiencia de Aprendizaje Mediada (EAM)

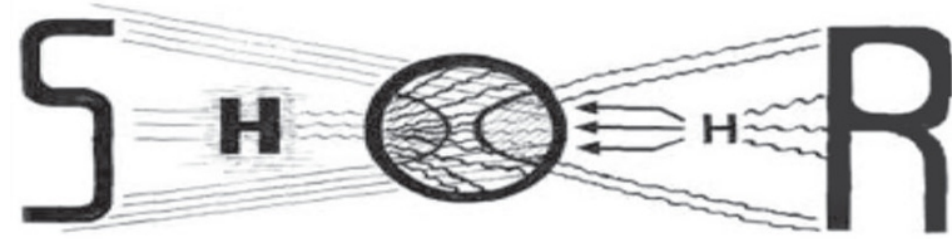

Fuente: Feuerstein, et. al. (1994)

Figura 2.

Modelos de aprendizaje

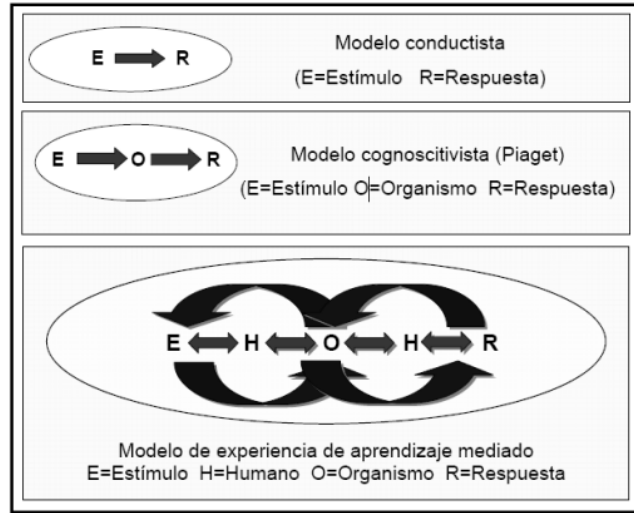


La figura 1 y 2 muestra tres modelos pedagógicos en donde el modelo de la EAM explica la función del acto humano mediador en el uso de estímulos y la inducción de la respuesta del organismo mediado. Describe Feuerstein $(1980$, p. 16) la relación entre EAM y la modalidad de exposición directa del aprendizaje de la siguiente forma: "el niño que más haya estado en condiciones de EAM y la más óptima en el proceso mediacional, mayor será la capacidad del niño para beneficiar y se modifican por la exposición directa a los estímulos". De este modo, la EAM permite formar al sujeto para que con posterioridad a la intervención pueda responder de manera efectiva y adecuada a los estímulos directos. Este tipo de planteamientos tienen una relación con la Zona de Desarrollo Próximo, planteada por Vygotsky (1985), la cual se define como:

La distancia entre el nivel real de desarrollo, determinado por la capacidad de resolver independientemente un problema, y el nivel de desarrollo potencial, determinado a través de la resolución de un problema bajo la guía de un adulto o en colaboración con un compañero más capaz. El estado del desarrollo mental de un niño puede determinarse únicamente sí se lleva a cabo una clasificación de sus dos niveles: del nivel real del desarrollo y de la zona de desarrollo potencial.

Al observarse los aportes de Vygotsky (1985) y los de Feuerstein, et. al. (1994) se encuentran dos postulados muy estrechamente relacionados por cuanto el primero considera que la Zona de Desarrollo Próximo es el nivel del niño(a) para realizar alguna actividad acompañado de una persona con mayor capacidad, lo cual para Feuerstein es el proceso de mediación como instrumento para generar nuevos estados en el sujeto.

La EAM constituye por lo tanto una concepción teórica que explica el vínculo y la interacción humana en la construcción de nuevos estados del sujeto y su objetivo es generar efectos sobre el organismo para modificar el pensamiento de acuerdo al criterio de autoplasticidad. La ausencia de este tipo de procesos tiene efectos negativos sobre las personas, quienes adquieren rigidez en sus procesos mentales. En la Figura 3 están representados los factores etiológicos distales y próximos del desarrollo cognitivo y relacionados con la mediación formulados por Feuerstein. Los factores etiológicos son determinantes del desarrollo cognitivo diferencial, en otras palabras, son determinantes para que el organismo tenga "formas bajas y niveles (...) inadecuados de funcionamiento cognitivo hasta niveles superiores de pensamiento" (Prieto, 1989).

Los factores etiológicos son de dos tipos: distales y próximos. Los primeros se refieren a los que proporciona el medio en que se desenvuelve el sujeto como la herencia/factores genéticos, factores orgánicos, nivel de maduración, equilibrio emocional del niño y de los padres, estímulos ambientales, status socio-económico/nivel educativo, diferencia cultural. Los factores etiológicos próximos están constituidos por la ausencia o existencia de experiencias de aprendizaje mediado. A través de la gráfica se explica la incidencia de los factores endógenos, endo-exógenos y exógenos que producen efectos sobre el desarrollo de las personas y que pueden ser tomados en el campo formativo. Estos son los factores etiológicos distales. En el mejor de los casos, se generan experiencias de aprendizaje mediado y en el otro caso hay carencia de dichas experiencias. A esto la teoría de la MEC identifica como determinantes próximos. Por lo tanto, los efectos de los factores etiológicos distales no son los únicos determinantes en el desarrollo cognitivo de los sujetos, condiciones que pueden ser modificadas mediante próximos relacionados con la existencia o carencia de experiencias de aprendizaje mediado. En este orden, Feuerstein y Rand (1974) consideran que ningún estado resulta irreversible por lo que puede ser modificado a menos que existan graves daños orgánicos genéticos. 
Figura 3.

Factores etiológicos asociados a la Mediación

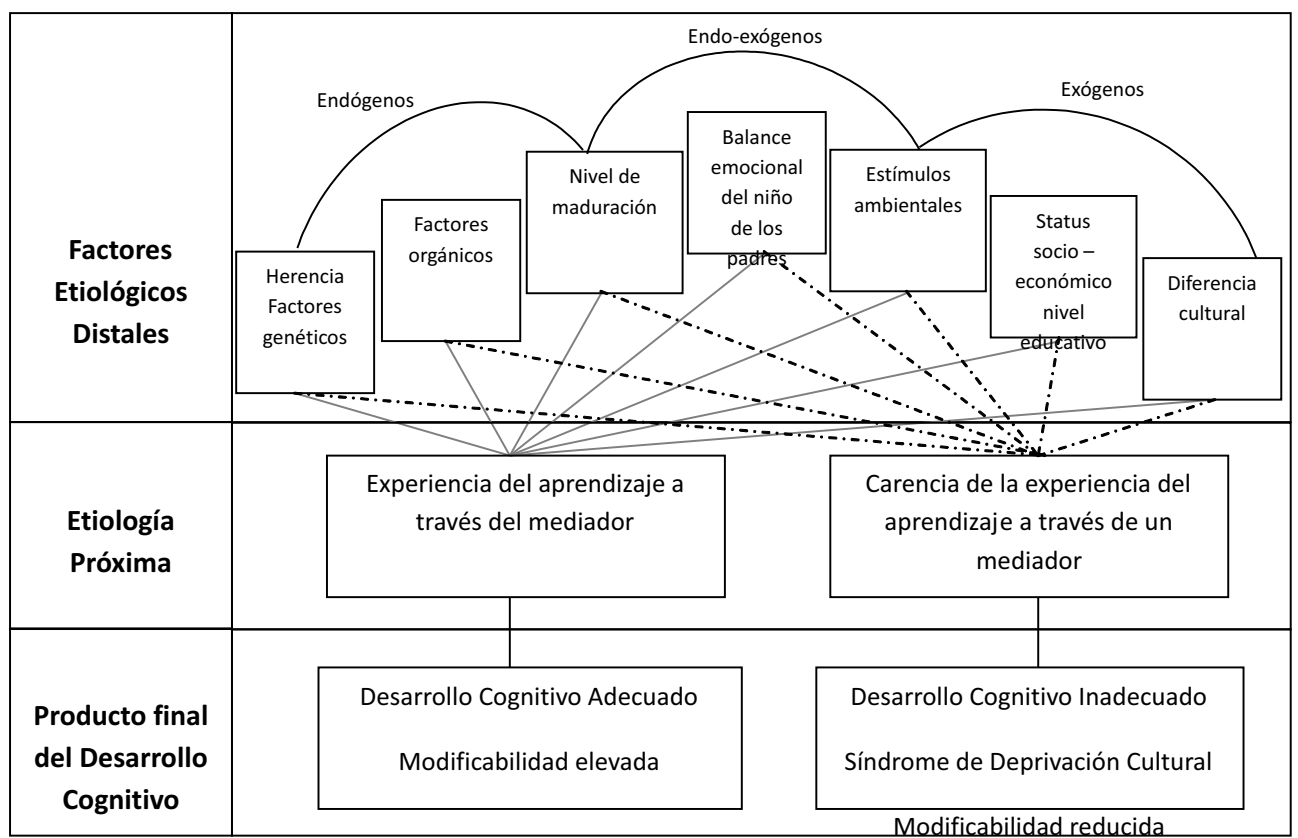

En síntesis, todos estos factores pueden incidir de manera positiva o negativa sobre el organismo pero no implica un estado definitivo en el individuo dependiendo básicamente de los estímulos y la calidad de los mismos sobre el organismo para propiciar la modificabilidad. De la misma manera, la EAM es necesaria para corregir las deficiencias cognitivas y potencializar las operaciones mentales. Para ello, la EAM, a través del PEI, trabaja sobre las funciones cognitivas, las cuales son definidas como las "actividades del sistema nervioso [que brindan] la capacidad al individuo para servirse de la experiencia previa en su adaptación a nuevas situaciones" (Feuerstein, et. al. 1997).

- Funciones cognitivas.

Las funciones cognitivas son prerrequisitos de la inteligencia para el adecuado tratamiento de la información y lograr la autorregulación del organismo (Feuerstein, et. al. 1997). Es así que las funciones cognitivas tienen relación con la adaptación de las personas a nuevas situaciones por cuanto hace uso de conocimientos previos para dicho fin. Piaget (2005, p. 19) entiende las funciones cognitivas como aquellas que van desde la percepción y las funciones sensorio-motrices hasta la inteligencia abstracta, incluidas las operaciones formales. Añade, que estas funciones cognitivas están relacionadas, y de una manera indisoluble, con las funciones afectivas, por lo tanto, los elementos cognitivos no serán desarrollados si no existen elementos afectivos y viceversa. 
Piaget explica que el organismo, frente a un nuevo objeto del conocimiento, incorpora el mismo en los esquemas conceptuales previos (asimilación) y después de ello el organismo se modifica (acomodación), lo cual presupone la adaptación, es decir, un sentimiento de satisfacción (Piaget, 2005. p. 20, 21). De esta manera, los aportes de Piaget son tomados por Feuerstein en su teoría: la acomodación junto con el sentimiento de satisfacción presupone lo que Feuerstein señaló como adaptación. Las funciones cognitivas pueden verse afectadas y exteriorizarse en el sujeto. Buela-Casl y Sierra (1997, p. 614) describe la función cognitiva como "el componente básico de la inteligencia" por lo que es un prerrequisito del acto de pensar inteligentemente. De la misma forma, estos autores señalan el procedimiento en los casos de deficiencias cognitivas según nivel, demostrando la aplicación de la teoría de la MEC y de la EAM en diferentes contextos de dificultad.

\section{Campos De Aplicación De La Teoría De La Mec.}

Los estudios e investigaciones en torno a la teoría de la MEC constituyen un importante aporte dentro de la validación de los principios teóricos que cimientan la teoría de Feuerstein. Algunos de estos trabajos han sido desarrollados en Latinoamérica y otros (la mayoria), en Estados Unidos y en Europa, localizados todos dentro de bases de datos científicas. Se pueden destacar en español los trabajos realizados por García y Porras (2000); Himmel, et. al. (1996); Velarde (2008); el trabajo a gran escala en Bahía (Brasil) realizado por Varela, et. al. (2006); y Cerrillo (2001). En inglés resultan significativos los estudios de Feuerstein, et. al. (1981); Tzuriel y Kaufman (1999); Tzuriel y Weiss (1998); Lifshitz y Rand (1999); Embretson (1992); Tzuriel y Caspi (1992); Tzuriel y Shamir (2007); Portowitz et. al. (2009); Carl, et. al. (1985); Skuy, et. al. (1992); Kozulin, et. al. (2010); Savell, et. al. (1986).

García y Porras (2000) a través de un estudio experimental aplicaron el PEI a estudiantes en edades de 6 a 9 años del grado primero del primer ciclo. Abordaron las diversas críticas hechas a los programas de Training Cognitivo (entrenamientos para el desarrollo del pensamiento o la inteligencia): a) la exclusión de factores sociales y éticos, y sobredimensión de la perspectiva psicológica respecto de la enseñanza; b) no toma en cuenta el contexto sociohistórico y cultural de la población, además de los contenidos curriculares que no son abordados dentro del desarrollo de los programas; c) la rigidez en la ejecución de los programas para mediadores y mediados restringiendo la libertad metodológica y pedagógica; d) insuficiencia de los estudios al intentar explicar el impacto del programa sobre la población respecto de las habilidades entrenadas o los beneficios adquiridos a largo plazo; y e) la validación de los programas por errores en el diseño metodológico.

A través de los resultados obtenidos a partir del desarrollo del trabajo se superan algunas de las críticas identificadas por los autores García y Porras (2000). De estos cabe destacar los siguientes:

a) Mejoramiento del desempeño del área de matemáticas a partir del desarrollo del Instrumento de Relaciones Espaciales.

b) Creación de hábitos de trabajo en las diferentes áreas.

c) Conformación de equipos y buen ambiente de trabajo en ellos.

En esta investigación se alcanza a inducir un impacto de tipo cualitativo en matemáticas y en la intencionalidad de los sujetos abordados frente a los procesos académicos. Por su parte, Himmel et. al. (1996) realizaron un estudio con niños y niñas de la misma edad (transición 
mayor) intentando establecer las relaciones entre PEI y las áreas de matemáticas, lectura y escritura. A partir de la aplicación del PEI se obtuvo un mayor acercamiento entre mediador y estudiante, mejoramiento de la capacidad verbal y corrección de funciones cognitivas deficientes.

En este sentido es necesario anotar, que como se señaló en las críticas recopiladas por García y Porras (2000), los resultados no corresponden a la intención planteada como objetivo general, por cuanto no se observa la relación entre el PEI y el mejoramiento en las matemáticas, la lectura y la escritura. Para un correcto uso del PEI y superar las dificultades presentadas con los programas de training cognitivo, García y Porras (2000), sugieren que se deben considerar los siguientes como requerimientos el lugar de trabajo, el papel del tutor, relación directa de los instrumentos del PEI con los contenidos curriculares, entre otras.

De manera contraria a Himmel et al. (1996), Cerrillo (2001) logra establecer mejoras en el rendimiento académico en los estudiantes acudiendo a los programas cognitivos como el de Feuerstein o Harvard (Programa CORAL) sobre jóvenes de $2^{\circ}$ curso de Educación Secundaria Obligatoria (ESO) en Madrid, (España). A partir de un pre-test y un pos-test se alcanzó potenciar: a) el razonamiento lógico; b) la inteligencia en general; c) la inteligencia no verbal; d) el auto concepto académico; e) el mejoramiento en las pruebas de lengua y matemáticas en cerca de 20 puntos respecto de la prueba inicial. Los resultados de Cerrillo (2001) son más contundentes por cuanto es medible en aspectos específicos de la inteligencia lo cual permite determinar la viabilidad del uso del PEI y de la EAM.

Velarde (2008) aborda otro tipo de población respecto de las investigaciones consultadas: la rural y en edades de 8 a 10 años. La muestra estuvo determinada por 47 estudiantes ( 25 del grupo experimental y 22 de del grupo de control) del grado tercero de básica primaria de una escuela en Murcia (España). El autor aplicó dos pruebas de inteligencia al iniciar el proceso y al finalizar (Test Wechsler -WISC- y Test de Matrices Progresivas de Raven). Como parte de sus resultados se encuentra la validación de la hipótesis, es decir, que hubo mejoramiento cognitivo y la modificación de las estructuras del pensamiento en población con deprivación cultural. De la misma manera, plantea un cambio de actitud de los docentes frente al proceso de enseñanza y aprendizaje con los estudiantes y se logra integrar el PEI con el diseño curricular de la institución.

Como experiencia a gran escala, se encuentra el trabajo realizado en Bahía (Brasil). Varela, et. al. (2006) desde el año 1999 hasta el momento han implementado el PEI como parte de la política curricular y los resultados han sido sobresalientes. A partir de su inicio, se ha pasado de una cobertura desde 1999 hasta el 2005 de 15.580 a 767.490 estudiantes, de 472 a 6.416 profesoras, de 18 a 280 escuelas y de 17 a 91 municipios.

Como parte de la implementación del PEI en los diferentes niveles, se han obtenido como resultados (i) la mejora en funciones báscias como la observación, la identificación, la localización, la descripción, la representación, etc., en 84\% de la población; (ii) la mejora en operaciones mentales como la clasificación, la seriación, el análisis, la interpretación, entre otras, en un $63 \%$ de la población.

Ahora bien, en cuanto a los trabajos desarrollados en otros espacios diferentes al latinoamericano, inscriben la TMEC dentro del campo de los niños y niñas con dificultades de aprendizaje y bajo rendimiento. En efecto, muchas de estas contribuciones investigativas 
están publicadas en revistas que abordan temas sobre la educación especial, cultura, desarrollo, retardo mental, currículo, etc., y en diferentes contextos como de inclusión, relaciones familiares, incapacidad para un aprendizaje adecuado, educación para adultos, entre otros. Uno de los principales autores en el campo de la aplicación de la TMEC es su mismo creador, Reuven Feuerstein. Son numerosos sus estudios en torno al desarrollo de la teoría. Un ejemplo, es el estudio titulado Modificabilidad Cognitiva en la adolescencia: estructura cognitiva y los efectos de intervención, (Feuerstein, et. al., 1981) en donde describe que el PEI junto con el EAM permite una intervención que remedia el fenómeno del bajo rendimiento cognitivo, pues sensibiliza al sujeto frente a las fuentes formales e informales de información.

Se han desarrollado otros estudios que abordan la cultura de los sujetos de aprendizaje en relación con la modificabilidad cognitiva y la EAM. Tzuriel y Kaufman (1999) examinan el caso de los niños inmigrantes etíopes que han llegado a la sociedad israelí. Para ello, utilizaron una evaluación dinámica basada en los conceptos de zona de desarrollo próximo de Vygotsky y la EAM de Feuerstein, para posteriormente aplicar un proceso de enseñanza corto pero intenso, en donde los resultados evidenciaron una disminución de las brechas entre los dos grupos.

Otros estudios como Tzuriel y Weiss (1998) analizan los efectos de la aplicación de la EAM en las interacciones madre-hijo, la aceptación, el rechazo y la predicción de la modificabilidad. Para ello se utilizaron juegos libres y estructurados en donde se grabaron 26 niñas y 28 niños del grado segundo. La matriz de análisis fue formulada de acuerdo a cinco criterios de la mediación de la TMEC: intencionalidad y reciprocidad, trascendencia, significado, sentimientos de competencia y la regulación de la conducta. Del mismo modo, se utilizó un cuestionario para evaluar la aceptación o el rechazo de los padres sobre la personalidad de los niños. Los resultaros evidenciaron que los criterios utilizados para la mediación influyeron en los factores distales, y por ende, en la aceptación o rechazo de las madres respecto de la personalidad de sus hijos.

También se han realizado estudios sobre el impacto del PEI en adultos con retraso mental. Lifshitz y Rand (1999) analizaron en 71 adultos divididos en cuatro grupos la aplicación del PEI en relación con el pensamiento lógico, el pensamiento predictivo y el pensamiento profundo, a través de un pre-test y un post-test. En todos los casos se encontró una mejoría significativa aunque en el caso de los adultos con sindrome de Down los resultados fueron inferiores frente a los adultos con otro tipo de discapacidad mental.

Un grupo con dificultades también abordado para la validación de la TMEC son los niños preescolares sordos, cuyos resultados se compararon con niños oyentes (Tzuriel y Caspi, 1992). La muestra estuvo conformada por 26 personas sordas y 26 oyentes en edades de 4, 1 y 6,3 años a quienes se les aplico la Matriz Progresiva Coloreada de Raven (RCPM), la prueba de Modificabilidad del Pensamiento Analógico para la Infancia (CATM), la Prueba de Analogía Pictórica (PAT) y el subtest de Analogías de la Prueba de Inteligencia SnijdersOomen-verbal (SON). En los resultados del RCPM, SON y PAT no se encontraron diferencias. En el CATM, los niños con incapacidad obtuvieron resultados inferiores que los niños oyentes, sin embargo, sus resultados mejoraron cuando se aplicó el PEI y la EAM.

Otro grupo abordado a través de la TMEC y del PEI son niños con problemas mentales (DCD, $\mathrm{CP}$, deterioro intelectual de origen genético, el trastorno del espectro autista, TDAH y otros trastornos del aprendizaje) de cinco a siete años de Canadá, Chile, Bélgica, Italia e Israel. E1

[at

AGO.USB Medellín-Colombia $\quad$ V. $13 \quad N^{\circ} 2$ PP. 279- 539 Julio-Diciembre 2013 ISSN: 1657-8031 
programa fue implementado a 104 niños en un periodo de 30 a 45 semanas con tiempos que oscilaron entre 27 y 90 horas. Los resultados obtenidos demostraron una mejora en el funcionamiento cognitivo de los niños, con mayores beneficios en aquellos casos donde todos los profesores se encontraban comprometidos con los principios de la EAM.

En otro escenario se evalúo el impacto de la EAM en niños en edad escolar (primero y tercero) en donde se diseño un programa de formación para que los niños de tercer grado sirvieran de pares frente a los niños de primer grado (Tzuriel, y Shamir, 2007). El modelo de mediación entre pares está basado en la teoría sociocultural de Vygostky y la EAM de Feuerstein y el objetivo en el caso del estudio fue investigar los efectos de la mediación entre compañeros con niños pequeños. Participaron en el estudio 178 alumnos (89 mediadores en grado $3^{\circ}$ y 89 alumnos de los grados $1^{\circ}$ ). Los mediadores correspondientes al programa de modificabilidad cognitiva demostraron mejores resultados en las pruebas y tuvieron que hacer frente al nivel cognitivo bajo de sus pares, "facilitando su enfoque mediacional y por lo tanto mejorar el rendimiento del alumno".

En el campo de las artes también se han realizado estudios con el fin de verificar los resultados de la teoría de la $\mathrm{MEC}$ en los procesos de formación a través de un programa de música. En la investigación realizada por Portowitz, et. al. (2009) se tomaron niños de alto riesgo de una escuela primaria para evaluarse en tres componentes: a) el contenido de las clases de música, b) los marcos interactivos, educativos (mediadas por ambientes de aprendizaje), c) habilidades específicas de aprendizaje. La muestra estuvo conformada por 81 niños (46 de grupo experimental y 35 del grupo de control). Los resultados indicaron diferencias significativas entre los grupos en el desarrollo de las habilidades cognitivas específicas.

A partir de la TMEC en colaboración con las teorias de Piaget y Vygostky se han diseñado, implementado y evaluado currículos para niños pequeños en situación de riesgo como es el caso del estudio de Carl, et. al. (1985). En el estudio se elaboró un currículo basado en las teorias mediacionales y dirigidos a los maestros para colaborar en su planificación e instrucción. Los resultados "mostraron ganancias significativas en el desarrollo cognitivo general, después de un año escolar del plan de estudios".

Ampliando el espectro de la aplicación de la TMEC se encuentra el estudio de Skuy, et. al. (1992) en donde se implementó el Dispositivo de Evaluación del Potencial de Aprendizaje (DEPA) y luego la EAM en grupos de adolescencia con esquizofrenia. El número de la muestra correspondió a 12 adolescentes con esquizofrenia y 10 adolescentes con trastorno de conducta grave. Explica los autores que "los resultados son alentadores en tinte no había pruebas de la modificabilidad de los subgrupos experimentales, tanto de la esquizofrenia y el trastorno de conducta grupos".

Cabe mencionar, que hay otro tipo de estudios en los que se aborda las dificultades para validar los resultados de la modificabilidad estructural cognitiva, aunque también se aborda nuevos desarrollos que permiten resolver estos problemas de validación lo cual resulta ser compatible (el aprendizaje de determinado conocimiento y el constructo -TMEC-) (Embretson, 1992). En efecto, Savell, et. al. (1986) menciona que los métodos aplicados de la teoría de Feuerstein son validos después de analizarse los resultados de investigaciones empíricas desarrolladas al respecto. Estos trabajos (los relacionados con anterioridad) constituyen los aportes investigativos al objeto de estudio que aborda el proyecto. Se ha 
podido establecer, a manera de conclusión, la efectividad del PEI y de la EAM de la TMEC de Feuerstein. Sin embargo, considero, en cierto sentido, que los efectos del programa van más allá de los determinados en los resultados de estas investigaciones. Esta consideración surge de la relación existente entre MEC y Desarrollo Humano.

Otro aspecto relevante del análisis de las investigaciones está centrado en el trabajo realizado con diferentes grupos poblacionales con dificultades de diverso origen y en donde la validación del PEI y de la EAM de la TMEC son referencias especificas del importante campo de aplicación que tiene las contribuciones de Feuerstein.

\section{Conclusiones.}

La teoría de la MEC y los sistemas elaborados a partir de estos (el PEI, el DEPA y los Ambientes Modificantes) son instrumentos de aplicación en aquellos casos donde el desarrollo humano se encuentra comprometido. El auge y la validez de los aportes de Feuerstein son muy valiosos aún más en un contexto de crisis y de exclusión permanente. El breve análisis de las investigaciones realizadas en torno al tema de la teoría de la MEC y de su aplicación en diversos contextos son un argumento para sostener el gran impacto de la posibilidad de cambio en miles de niños, niñas, jóvenes y adultos alrededor del mundo. Al respecto, se debe destacar que la aplicación de la teoría es altamente significativa de sobremanera en los grupos poblacionales que tienen serias dificultades. La deprivación cultural y su disminución en todas las personas a quienes se les ha aplicado la EAM o el PEI es una evidencia de los alcances de la teoría.

Los principios de la teoría de la MEC se evidencian en los múltiples estudios realizados y en la concordancia que tiene con otros postulados teóricos aceptados dentro de la comunidad científica. La edificación de la teoría de la MEC ha tomado los aportes de reconocidos teóricos como Piaget y Vygostky, y de la misma forma ha sustentado sus principios con los últimos adelantos de la neurociencia cognitiva. Su formulación es una contribución al campo del desarrollo humano, pues genera dos significativas ideas: primero, el organismo propenso a la modificación, es decir, hay sentido en todo procesos de formación; y segundo, la labor del maestro y otros profesionales vinculados al escenario del desarrollo humano. 


\section{Referencias Bibliográficas.}

Ausubel, D. (1976). Significado y aprendizaje significativo. En: Ausubel, D. (1976); Psicología educativa: un punto de vista cognoscitivo. México: Editorial Trillas. p.p. 55-107.

Buela-Casal, G. \& Sierra, C. (1997). Manual de evaluación psicológica: fundamentos, técnicas y aplicaciones. Madrid: Siglo XXI. 1056 p.

Carl, H.; Brooks, P. \& Burns, S. (1985). Stimulating Cognitive Development at Developmental Level: A Tested, Non-Remedial Preschool Curriculum for Preschoolers and Older Retarded Children. Special Services in the Schools, Vol. 3 (1-2). p.p. 127-147.

Cerrillo, M. (2001). Atención a la diversidad por medio de un programa de intervención sociocognitiva. Tendencias Pedagógicas, No 6. p.p. 187-199.

De Zubiría, J. (1997). Tratado de pedagogía conceptual: Los modelos pedagógicos. Bogotá: Fondo de Publicaciones Bernardo Herrera Meriño. 160 p.

Embretson, S. (1992). Measuring and Validating Cognitive Modifiability as an Ability: A Study in the Spatial Domain. Journal of Educational Measurement, Vol. 29 (1). p.p. 25-50.

Feuerstein, R. (1963). Children of the Melah. Socio-cultural deprivation and its educational significance. Israel.

Feuerstein, R. (1980). Instrumental Enrichment: An intervention program for cognitive modifiability. Baltimore: University Park Press. 436 p.

Feuerstein, R. (1991). Entrevista realizada por la periodista de los Ángeles Covarrubias Claro, El Mercurio, Domingo 22 de Diciembre. Santiago de Chile.

Feuerstein, R.; Klein, P. \& Tannenbaum, A. (1994). Mediated Learning Experience (MLE): theoretical, psychosocial and learning implications. Israel: Freund Publishing House Ltd. $391 \mathrm{p}$.

Feuerstein, R. \& Lewin-Benham, A. (2012). What Learning Looks Like: Mediated Learning in Theory and Practice, K-6. Nueva York: Teachers College Press. 219 p.

Feuerstein, R.; Miller, R.; Hoffman, M.; Rand, Y.; Mintzker, Y. \& Reimer Jensen, M. (1981). Cognitive Modifiability in Adolescence: Cognitive Structure and the Effects of Intervention. The Journal of Special Education, Vol. 15, (2). p.p. 269-287.

Feuerstein, R.; Naegelé, B.; Pépin, J. \& Lévy, P. (1997). Frontal lobe-related cognitive functions in patients with sleep apnea syndrome before and after treatment. Acta Neurológica Bélgica, Vol. 97 (2). p.p. 96-107.

Feuerstein, R.; Rand, Y. \& Rynders, J. (1988). Don't accept me as I am: Helping "retarded" people to excel. New York: Plenum Press. 322 p.

Feuerstein, R. \& Kozulin, A. (1995). The Bell Curve: Getting the facts straight. Educational Leadership, Vol. 52 (7). p.p. 71-74. 
Feuerstein, R. \& Rand, Y. (1974). Mediated Learning Experiences: An outline of the proximal etiology for differential development of cognitive functions. International Understanding, No. 74. p.p. 7-36.

García, I. \& Porras, R. (2000). Condiciones para la aplicación de la adaptación del Programa de Enriquecimiento Instrumental (PEI) de Feuerstein en el primer ciclo de educación primaria. Kikirikí, No. 41. Recuperado el 13/05/2012 de:

http://www.quadernsdigitals.net/index.php?accionMenu=hemeroteca.VisualizaArt iculoIU.visualiza\&articulo_id=1169.

Gardner, H. (1985). Inteligencias múltiples. La teoría en la práctica. Barcelona: Paidós. 309 p.

Haywood, H. \& Tzuriel, D. (1992). Interactive Assessment. New York: Springer-Verlag. 533 p.

Himmel, E.; Álvarez-Salamanca, E.; Díaz, C.; Fuentes, S. \& Villalón, M. (1996). Funciones cognitivas y habilidades instrumentales necesarias para los aprendizajes formales en el niño. International Journal of Early Childhood, Vol. 28 82). p.p. 15-20.

Hobbs, N. (1980). Feuerstein's instrumental Enrichment; teaching intelligence to adolescents. Educational Leadership, Vol. 37 (7). p.p. 566-568.

Jackson, Y. y Feuerstein, R. (2011). The pedagogy of confidence. New York: Teachers College Press. 194 p.

Kozulin, A.; Gindis, B.; Ageyev, V. \& Miller, S. (2003). Vygostky's educational theory in cultural context. New York: Cambridge University Press. 479 p.

Kozulin, A.; Lebeerb, J.; Madella-Nojac, A.; Gonzalez, F; Jeffreye, I.; Rosenthalf, N. \& Koslowskyg, M. (2010). Cognitive modifiability of children with developmental disabilities: A multicentre study using Feuerstein's Instrumental Enrichment-Basic program. Research in Developmental Disabilities, Vol. 31 (2). p.p. 551-559.

Lifshitz, H. \& Rand, Y. (1999). Cognitive modifiability in adult and older people with mental retardation. Mental Retardation, Vol. 37 (2). p.p. 125-138.

Monclús, A. (2004). Educación y cruce de culturas. México: Fondo de Cultura Económica. $191 \mathrm{p}$.

Myers, D. (2005). Psicología. Buenos Aires: Medica Panamericana. 980 p.

Noguez, S. (2002). El desarrollo del potencial de aprendizaje. Entrevista a Reuven Feuerstein. Revista Electrónica de Investigación Educativa, Vol. 4 (2). Recuperado el 13/05/2012 de:

http://redie.ens.uabc.mx/vol4no2/contenido-noguez.html.

Orrú, S. (2003). Reuven Feuerstein y la teoría de la Modificabilidad Cognitiva Estructural. Revista de Educación, No. 332. p.p. 33-54. 
Piaget, J. (1980). Adaptación vital y psicología de la inteligencia. Madrid: Siglo XXI. 195 p.

Piaget, J. (2005). Inteligencia y afectividad. Buenos Aires: Aique.

Piaget, J. e Inhelder, B. (2007). Psicología del niño. Madrid: Morata. 161 p.

Pilonieta, G. (2003). La teoria de la Modificabilidad Estructural Cognitiva y su influencia en la pedagogía para el desarrollo de las inteligencias y las comunidades de aprendizaje. Asociación Colombiana para el Avance de las Ciencias del Comportamiento (ABA Colombia). Recuperado el 20/04/2011 de: www.abacolombia.org.com.

Pilonieta, G. (2004). Aventuras para el desarrollo de la inteligencia: la magia de la aventura. Universitas Odontológicas, No 24 (54/55). p.p. 103-106.

Pilonieta, G. (2010). Modificabilidad Estructural Cognitiva y Educación. Bogotá: Magisterio. $274 \mathrm{p}$.

Portowitz, A.; Lichtenstein, O.; Egorova, L. \& Brand, E. (2009). Underlying mechanisms linking music education and cognitive modifiability. Research Studies in Music Education, Vol. 31 (2). p.p. 107-128.

Prieto, J. (2000). Sócrates (p.p. 185-212). En: Fundación Canaria Orotava de Historia de la Ciencia (2000). Ciencia y cultura en la Grecia Antigua, Clásica y Helenística: Actas años VI y VII. Las Palmas de Gran Canaria, Canarias: Dirección General de Ordenación e Innovación Educativa. 405 p.

Prieto, M. (1989). Modificabilidad Cognitiva y PEI. Madrid: Bruño. 350 p.

Ramírez, S. y Roa, J. (2003). El Programa de Enriquecimiento Instrumental de Feuerstein: Una aproximación teórica. Revista Euphoros, No 6.

Savell, M.; Twohig, P. y Rachford, D. (1986). Empirical Status of Feuerstein's "Instrumental Enrichment" (FIE) Technique as a Method of Teaching Thinking Skills. Review of Educational Research, Vol. 56 (4). p.p. 381-409.

Skuy, M.; Apteer, A.; Dembo, Y.; Tyano, S.; Haniel, S. \& Tzuriel, D. (1992). Cognitive Modifiability of Adolescents with Schizophrenia: a Research Note. Journal of Child Psychology and Psychiatry, Vol. 33 (3). p.p. 583-589.

Suazo, S. (2006). Inteligencias Múltiples: Manual práctico para el nivel elemental. San Juan: Universidad de Puerto Rico. 304 p.

Tzuriel, D. (2001). Dynamic Assessment of Young Children. Nueva York: Kluwer Academic Plenum Plubishers. 241 p.

Tzuriel, D. y Caspi, N. (1992). Cognitive Modifiability and Cognitive Performance of Deaf and Hearing Preschool Children. The Journal of Special Education, Vol. 26 (3). p.p. 235252.

Tzuriel, D. y Kaufman, R. (1999). Mediated Learning and Cognitive Modifiability: Dynamic Assessment of Young Ethiopian Immigrant Children to Israel. Journal of CrossCultural Psychology, Vol. 30 (3). p.p. 359-380. 
Tzuriel, D. \& Shamir, A. (2007). The effects of Peer Mediation with Young Children (PMYC) on children's cognitive modifiability. British Journal of Educational Psychology, Vol. 77 (1). p.p. 143-165.

Tzuriel, D. \& Weiss, S. (1998). Cognitive modifiability as a function of mother-child mediated learning strategies, mothers' acceptance-rejection, and children's personality. Early Development and Parenting: an international journal of research and practice, Vol. 7 (2). p.p. 79-100.

Varela, A.; Gramacho, A. \& Melo, C. (2006). Programa de Enriquecimiento Instrumental (PEI): alternativa pedagógica que responde al desafio de calidad en educación. Diversitas, Vol. 2 (2). p.p. 297-310.

Velarde, E. (2008). La teoría de la Modificabilidad Estructural Cognitiva de Reuven Feuerstein. Investigación Educativa, Vol. 12 (22). p.p. $203-221$.

Vygotsky, L. S. (1985). Pensamiento y lenguaje. Buenos Aires: Pléyade. 429 p.

Wood, D. (2000). Como piensan y aprenden los niños. México: Siglo XXI Editores. 371 p. 